TABLE 2

The Hardy-Littlewood Constants

$$
\begin{aligned}
h_{-10} & =0.67111392 \\
h_{-9} & =0 \\
h_{-8} & =1.85005441 \\
h_{-7} & =0.75737123 \\
h_{-6} & =1.03575587 \\
h_{-5} & =1.77330507 \\
h_{-4} & =0
\end{aligned}
$$

$$
\begin{aligned}
h_{-3} & =1.38342429 \\
h_{-2} & =1.85005441 \\
h_{-1} & =0 \\
h_{0} & =0 \\
h_{1} & =1.37281346 \\
h_{2} & =0.71306310 \\
h_{3} & =1.12073275
\end{aligned}
$$

$$
\begin{aligned}
h_{4} & =1.37281346 \\
h_{5} & =0.52824557 \\
h_{6} & =0.71304162 \\
h_{7} & =1.97304317 \\
h_{8} & =0.71306310 \\
h_{9} & =0.91520897 \\
h_{10} & =1.08240211
\end{aligned}
$$

From Table 1, in turn, we may compute [3], [4] the Hardy-Littlewood constants $h_{a}$ for $a= \pm 5$ and \pm 10 . Together with previously computed values, we may thus complete an 8D table of $h_{a}$ for $a=-10(1) 10$ except for $a= \pm 7$. The $L_{ \pm 7}(s)$, needed to fill this gap, may also be expressed in terms of $I_{s}(\alpha)$ and $R_{s}(\alpha)$, but this time the arguments $\alpha$ are not given explicitly in [2], and elaborate interpolation would be required to obtain comparable precision.

Alternatively, as is known, generalized harmonic series, including $L_{a}(s)$ for integer $s$, may be expressed in terms of the polygamma functions [5], [6]. However, the same difficulty arises for $L_{ \pm 7}(s)$, and again elaborate and laborious interpolation is necessary. At the author's request John W. Wrench, Jr. has kindly computed $L_{7}(2), L_{7}(4), L_{-7}(3)$ and $L_{-7}(5)$ in this way, and these numbers, together with the closed-form $L_{ \pm 7}(s)$, suffice to complete our tabulation of $h_{a}$. This is given in Table 2.

Applied Mathematics Laboratory

David Taylor Model Basin

Washington, D. C. 20007

1. Daniel Shanks \& John W. Wrench, JR., "The calculation of certain Dirichlet series," Math. Comp., v. 17, 1963, p. 136-154; Corrigenda, ibid. p. 488.

2. Staff of the Computation Department, Mathematisch Centrum, Amsterdam, Polylogarithms, Report R24, Part I: Numerical Values, 1954. Reviewed in MTAC, v. 9, 1955, p. 40, UMT 29.

3. Daniel Shanks, "On the conjecture of Hardy \& Littlewood concerning the number of primes of the form $n^{2}+a, "$ Math. Comp., v. 14, 1960, p. 321-332.

4. Daniel Shanks, "Supplementary data and remarks concerning a Hardy-Littlewood conjecture," Math. Comp., v. 17, 1963, p. 188-193.

5. Harold T. Davis, Tables of the Higher Mathematical Functions, vol. 2, Principia Press, Bloomington, Indiana, 1935, p. 14.

6. Eleanor Pairman, "Tables of the digamma and trigamma functions," Tracts for Computers, No. I, Cambridge University Press, 1954.

\title{
New Factors of Fermat Numbers
}

\section{By Claude P. Wrathall}

Eleven new factors of Fermat numbers $F_{m}=2^{2^{m}}+1$ are listed below. A summary of the present status of the sequence $F_{m}$ is presented in Table 2.

The method used was suggested by Dr. J. L. Selfridge. Simply stated, the method consisted of forming a sieve array to eliminate possible factors divisible by a prime $\leqq 499$. The remaining possible factors were tested to determine if any of the congruence relationships

Received August 22, 1963, revised October 2, 1963. 
TABle 1

Factors $k \cdot 2^{n}+1$ of Fermat Numbers $F_{m}$

\begin{tabular}{c|l|l||r|r|l}
\hline$k$ & $n$ & $m$ & $k$ & $n$ & $m$ \\
\cline { 2 - 3 } 308385 & 21 & 19 & 149041 & 32 & 30 \\
534689 & 23 & 21 & 127589 & 33 & 30 \\
48413 & 29 & 25 & 1479 & 34 & 32 \\
143165 & 29 & 26 & 2653 & 40 & 38 \\
141015 & 30 & 27 & 43485 & 45 & 42 \\
& & & 4119 & 54 & 52 \\
\hline
\end{tabular}

TABLE 2

\begin{tabular}{|c|c|}
\hline$m$ & Character of $F_{m}$ \\
\hline $\begin{array}{l}0,1,2,3,4 \\
5,6 \\
10,11,12^{*}, 19,30,38 \\
9,15,16,18,21,23,25,26,27,32,36 \\
\quad 39,42,52,55,58,63,73,77,81,117 \\
\quad 125,144,150,207,226,228,250,267 \\
\quad 268,284,316,452,1945 \\
7,8,13,14 \\
17,20,22,24,28,29,31 \text {, etc. }\end{array}$ & $\begin{array}{l}\text { Prime } \\
\text { Composite and completely factored } \\
\text { Two or three* prime factors known } \\
\text { Only one prime factor known }\end{array}$ \\
\hline
\end{tabular}

$$
2^{2^{m}} \equiv-1 \bmod \left(k \cdot 2^{n}+1\right) \quad m=7,8, \cdots, n-2
$$

were satisfied.

The search program was executed on the IBM 709 at the University of Washington and on the IBM 7090 at the UCLA Computing Facility. All factors have been checked using the SWAC programs of Robinson [1].

For references relevant to Table 2 see Robinson [1], the references cited there, and the more recent papers [2] through [5].

Boeing Company

Airplane Division

Renton, Washington

1. Raphael M. Robinson, "A report on primes of the form $k \cdot 2^{n}+1$ and on factors of Fermat numbers," Proc. Amer. Math. Soc., v. 9, 1958, p. 673-681.

2. G. A. PAXson, "The compositeness of the thirteenth Fermat number," Math. Comp., v. 15,1961, p. 420 .

3. J. L. Selfridge \& Alexander Hurwitz, "Fermat numbers and Mersenne numbers," Math. Comp., v. 18, 1964, p. 146-148.

4. Hans Riesel, "A factor of the Fermat number F19," Math. Comp., v. 17, 1963, p. 458.

5. John Brillhart, "Some miscellaneous factorizations," Math. Comp., v. 17, 1963, p. 449 , Equation (29). 